\title{
Occupancy model of perceived numerosity
}

\author{
JÜRI ALLIK and TIIA TUULMETS \\ University of Tartu, Tartu, Estonia
}

\begin{abstract}
Observers saw 234 different pairs of stochastically organized dot patterns and indicated which of the two patterns appeared to be more numerous. All of the data can be accounted for by supposing that the choice of the more numerous pattern is based on the determination of the occupancy indices of both patterns. Each dot is posited to have an impact upon its neighborhood in a constant occupancy radius $R$. The area of the stimulus plane occupied collectively by all dots provides a basis for judging relative numerosity; the pattern with the larger occupancy value is chosen as more numerous. The occupancy model, besides providing a general explanation of known numerosity illusions in strictly quantitative terms, can explain some puzzling aspects of numerosity perception.
\end{abstract}

Quantification is one of the most impressive acts of the human mind. On many occasions, however, the direct one-by-one counting of items is impossible: the number of objects is too large, the viewing time is too limited, the separation of already-counted objects from not-yetcounted ones is too difficult, and so forth. Nevertheless, in all such situations, the observer is able to estimate the approximate number of items on the basis of an instantaneous impression of numerosity. In a typical numerosity discrimination experiment, the observer indicates which of the two presented random-dot patterns appears to be more numerous. It is intuitively compelling to think that the observer's decision is based on an internal representation of numerosity-that the pattern producing the greater subjective magnitude of an internal process is chosen as being more numerous. All currently constructed psychophysical scales describe perceived numerosity as a power function of the objective number of items in the stimulus (Indow \& Ida, 1977; Krueger, 1972, 1984). All these numerosity scales are very tentative, however, for they fail to take into account the well-documented dependence of perceived numerosity on the spatial configuration of dots. The same numbers of dots distributed differently in space may appear to be very different in the apparent number of their elements. For example, objects occupying a more extended area on the display usually appear to contain more numerous elements (Bevan, Maier, \& Helson, 1963; Binet, 1890; Ponzo, 1928). Many other configurations of dots have been found to increase or decrease in their apparent visual number, relative to the same number of randomly distributed dots (Frith \& Frith, 1972; Ginsburg, 1976; Ginsburg \& Goldstein, 1987;

The authors thank Norman Ginsburg, Tarow Indow, and an anonymous reviewer for helpful comments on an earlier version of this article. The discussion of this research topic with Piet Vos has been very stimulating for many years. We are grateful to Enn Veldi for help in revising our English. We are extremely obliged to Lester Krueger for his valuable suggestions and editorial help. Correspondence concerning this article may be sent to Jüri Allik, Department of Psychology, University of Tartu, 78 Tiigi Street, Tartu, Estonia 202400 (U.S.S.R.).
Krueger, 1972; Taves, 1941; Vos, van Oeffelen, Tibosch, \& Allik, 1988). Such results indicate that the perceptual system is not able to abstract the number per se from all the other stimulus attributes (see Allik, 1989).

Gestalt categories of perceptual organization, such as spatial proximity, provide more realistic candidates for the stimulus properties that serve as bases for numerosity judgments. Unfortunately, most of these principles have not been formulated in quantitatively measurable terms, and, as a rule, they can be communicated only through graphic examples. One of the few attempts to explain perceived numerosity in strictly formal terms was undertaken by Vos et al. (1988). The basic idea is that the perceived numerosity depends not on the number of dots as such but on a more complex spatial property of the dot pattern-namely, the total area of the plane apparently filled with dots. The impact each dot has upon its neighborhood is portrayed as a monotonically decreasing spread (dispersion) function. The regions in the image where the sum of all (potentially overlapping) individual spread functions exceeds a pre-established threshold value are regarded as being filled with dots.

A formal description of the CODE (COntour DEtector) model, including the selection of optimal parameters (form and width of the spread function, threshold value), was provided by van Oeffelen and Vos in 1983. According to the CODE model, the width of the spread function depends on the distance to its nearest neighbor. The filled area index predicted the sign or direction of many known numerosity illusions (Vos et al. 1988). In addition, five specially constructed 36 -dot patterns filling approximately $33 \%$ of the total area were rated as being more numerous than five 36-dot patterns filling only $15 \%$ of the total area. However, the model has not been tested in a more demanding manner. It is also worth noting that a general concern of the Vos et al. study, as with other numerosity studies in general, was to predict the sign of the numerosity illusion, not its magnitude.

Although the basic idea of the model-that the perceived numerosity can be identified with the filled area-seems 


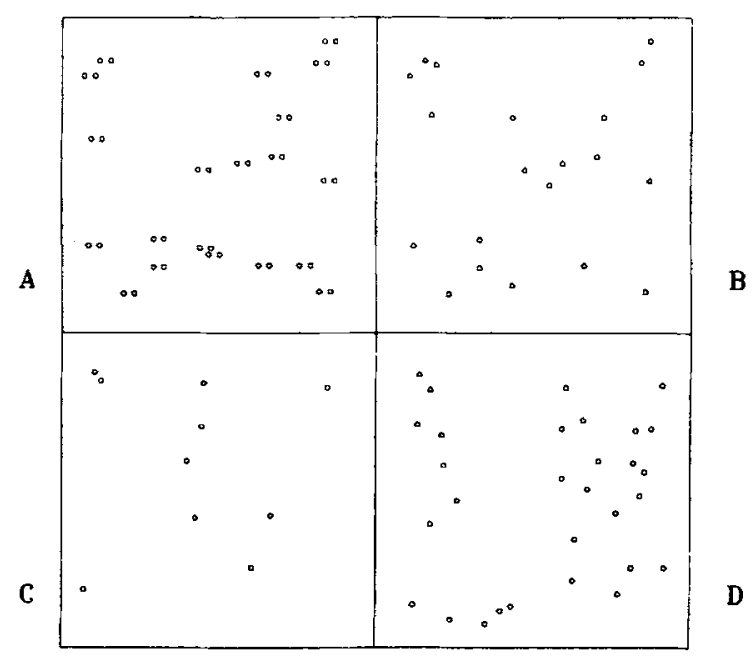

Figure 1. Four random-dot patterns whose perceived numerosities are incorrectly predicted by the CODE model. According to CODE, the 40-dot pattern, $A$, should appear to be the least numerous (filled area is $2.4 \%$ ), and the remaining three, $B, C$, and D, should appear to be about equally numerous (approximately $13 \%-14 \%$ of the stimulus area is filled) despite the various number of dots $(20,10$, and 30 dots, respectively).

to be excellent, it is easy to devise dot patterns whose perceived numerosity cannot be predicted by the CODE model, at least in its present form. In the CODE algorithm, the spread function shrinks as the distance to the nearest neighbor diminishes, collapsing to approximately zero in the extreme case when two dots occupy the two closest positions in space. Figure $1 \mathrm{~A}$ illustrates a pattern composed of 20 randomly distributed dots, each supplemented by a satellite dot shifted a few positions to the left or right.

According to the CODE algorithm, these 40 dots ought to appear to fill approximately $2.4 \%$ of the stimulus area, which is much less than the $13.3 \%$ filled by the 20 randomly distributed dots in Figure 1B. The filled area predictions are $13.0 \%$ and $13.8 \%$, respectively, for the other two random distributions of 10 (Figure 1C) and 30 (Figure 1D) dots. Despite a substantial difference between these two patterns, the CODE model predicts their perceived numerosity to be about equal. In fact, Pattern $C$ appears to be clearly less numerous than all the remaining patterns, and Pattern B, in its turn, appears to be less numerous than Patterns A and D.

These simple examples demonstrate two obvious weaknesses of the CODE model: First, it overemphasizes the nearest neighbor distance in the satellite patterns (Figure 1A), and, second, it is quite insensitive to large increases or decreases in the number of elements in the random patterns (Figures 1B-1D). Van Oeffelen and Vos (1983) stressed that since CODE considers only the relative proximity between dots, not their absolute distances, it is invariant under similarity transformations such as changes of size. Of course, the independence from the number of elements per square area may be a desirable property by itself, but certainly not for a model expected to explain perceived numerosity. From these observations, we can conclude that CODE cannot pretend to be a realistic model of perceived numerosity and that its success in the prediction of the sign of some numerosity illusions is first and foremost founded on a fortunate selection of stimulus material.

Ginsburg and Goldstein (1987) offered another set of spatial statistics with which to predict perceived numerosity. Divide the stimulus field into a large number of equally sized probe areas. The number of dots per probe area is a random variable with mean $\mu$ and variance $\sigma^{2}$. The ratio between the second and the first moments of this distribution, $\sigma^{2} / \mu$, is frequently used as an index of deviation from a completely random (Poisson) distribution of elements (Diggle, 1977; Ripley, 1981). If this ratio is smaller than that of a completely random distribution, the pattern appears to be more regular, and if the ratio is larger, the pattern appears to be more clustered. Ginsburg and Goldstein (1987) demonstrated that regular patterns were judged to be more numerous than random ones, and random patterns, in turn, more numerous than clustered ones. Although the ratio of clusteredness, $\sigma^{2} / \mu$, is associated with perceived numerosity somewhat, it is not suited in principle for discrimination of numerosity. Two patterns with a substantially different number of elements can have exactly the same value of $\sigma^{2} / \mu$, yet still be discriminated quite well (Ginsburg \& Goldstein devised patterns of 37, 74, and 111 elements that had identical indexes of clusteredness). Thus, although the dependence of perceived numerosity on spatial organization as indexed by the ratio $\sigma^{2} / \mu$ is an interesting empirical observation, it does not provide a full quantitative model of perceived numerosity. Also, it is rather doubtful that the mean number of dots per probe area $\mu$ (or any of its derivatives) is used as an index of numerosity, because $\mu$ is completely insensitive to the spatial configuration of elements.

Proposed model. Our aim in the present study is to propose a sufficiently general model of perceived numerosity. For that purpose, it is necessary, first, to have a broad variety of dot patterns, differing from one another both in the number and the spatial organization of their elements. There are many ways to produce dot patterns with different spatial organizations of elements. One of the simplest parameters characterizing a pattern of dots is the distance from each dot to its neighbors (Diggle, 1983; Ripley, 1981). In a completely random (Poisson) distribution, the expected number of neighbors within distance $t$ from a given dot is proportional to $t^{2}$ (Ripley, 1977; Schachter \& Ahuja, 1979).

The expected number of neighbors within radius $t$ can be either smaller or larger than $t^{2}$. In the first case, the dot appears to repel its neighbors as if it were an animal defending its territory (Schachter \& Ahuja, 1979). In the second case, the dot appears to attract some other dots, and, on the average, the nearest neighbor is closer than it would be in a completely uniform distribution. We used 
various generation processes to obtain both types of dot patterns, with neighbors closer or farther than would be expected in the case of a completely uniform distribution.

The next step after building such patterns is to determine the probability that each pattern will be judged as being more numerous than some other members of the set of patterns. Previous researchers have been content to demonstrate that two dot patterns equal in number but not in spatial arrangement of elements appear unequal in their perceived numerosity. In the present study, we will try to predict not only the sign of numerosity illusions, but also the precise degree of under- and overestimation of the numerosity.

Our goal, then, is to construct a formal model explaining how numerosity is perceived. By formal model, we mean a precisely specified property of dot patterns from which it is possible to predict every individual choice probability. This unknown stimulus property, although perfectly objective, may be relatively complex and does not typically figure in the list of quantities that statisticians use to describe two-dimensional point processes. It is even possible that such a single property does not exist at all, because the observer could, in principle, rely on one property in one situation and another property in another. To our surprise, we discovered that a relatively simple property of dot patterns, the occupancy index, accurately predicts all the observed individual choice probabilities.

\section{METHOD}

\section{Subjects}

Two observers, T.T. (29, female) and Y.S. (24, male), both with normal uncorrected vision, participated in this experiment. One of the subjects was naive, having no knowledge about the types of patterns used in this experiment.

\section{Stimulus Materials}

The patterns were generated and displayed on a screen with an LSI-11/03-compatible microcomputer. At a distance of $1 \mathrm{~m}$, the full display was approximately $13.3^{\circ}$ wide and $9.5^{\circ}$ high. Each of the possible stimulus dot locations on the graphic display was about $2^{\prime}$ of arc in size, and when each dot was lit, it had a luminance of $1.1 \mathrm{~cd} / \mathrm{m}^{2}$. Two random-dot patterns, confined within an invisible square of $120 \times 120$ pixels each and separated from each other by a 60 -pixel gap $\left(2.1^{\circ}\right)$, were presented in every single trial. The patterns were exposed for $400 \mathrm{msec}$ and then replaced by a blank field. This exposure time was chosen, on the basis of what had been found in a preliminary experiment, to be comfortable for the observer and sufficiently short to prevent direct counting. One of the two patterns was randomly chosen before each trial as a reference pattern. The experiment was divided into two separate sessions, and the number of reference dots was 20 in one session and 40 in the other. The distribution of dots in the reference pattern was random; the $x$ - and $y$-coordinates were obtained from a generator of random numbers, and if the specified location in the hemifield was not already occupied, a new dot was added to the pattern. Consequently, the location of each dot was independent of the location of all the other dots. The second of the two patterns was the test pattern. The number of dots in the test pattern relative to the number in the reference pattern was varied across nine levels: $-8,-6$, $-4,-2,0,2,4,6$, and 8 . There were four different twodimensional stochastic processes for test generation.

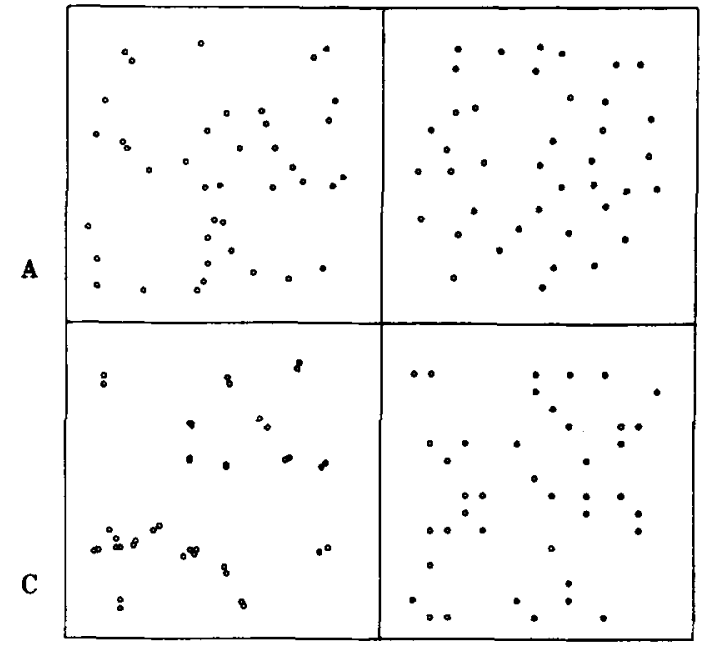

Figure 2. Example of four test pattern types used in the experiment: (A) random, (B) inhibition, (C) satellite, and (D) lattice.

Random process (Figure $2 \mathrm{~A}$ ). The $N$ dots were distributed randomly across the 14,400 possible positions in a hemifield. The principle of generation was the same as that of the reference pattern.

Inhibition process (Figure 2B). This process prohibited any two dots from being closer to each other than $d_{1}$ (inhibitory distance) pixels vertically or horizontally. Each dot was centered in a $D \times D$ pixel square, which no other dot could enter. The possible values of $d_{\mathrm{I}}$ were $2,4,6$, and 8 pixels.

Satellite process (Figure $2 \mathrm{C}$ ). Half of all the dots were randomly distributed in the plane. Each of the remaining N/2 satellite dots was assigned to its respective parent. The parent dot was centered in a $D \times D$ pixel square, and the position of the satellite dot within the square was randomly chosen. Consequently, every dot had at least one neighbor not more than $D / 2$ pixel away (the satellite distance $d_{\mathrm{s}}=8,16,24$, and 32 ) vertically or horizontally.

Lattice process (Figure 2D). A smaller set of possible positions was selected by means of a superimposed grid. The intersection of every $n$th column and every $n$th row provided a potential location. The number of possible locations decreases with lattice coarseness. An increase of the grid step $\left(d_{L}=2,4,6\right.$, and 8$)$ increases the minimal distance between the two nearest neighbors, increasing the empty territory around each dot and making the regular lattice structure more apparent.

\section{Procedure}

The observer was instructed to indicate which of the two patterns, the left or the right one, contained more dots. No feedback was provided. A new trial began a few seconds after the previous answer was given. Two experimental sessions, each with one of the two reference patterns $(N=20, N=40)$, were carried out one after the other. Both sessions were divided into 20 smaller successive sessions, within each of which all 117 different test patterns $(9$ random +36 inhibition +36 satellite +36 lattice) were presented in a completely random order. During the subsession, each test pattern type was displayed 5 times, and only random patterns were presented 20 times each. In total, the subject proceeded through 29,200 single trials on the basis of which 234 different test pattern choice probabilities were determined.

\section{RESULTS}

Figure 3 shows the test pattern choice probability in standard normal deviates or $z$ scores as a function of the 

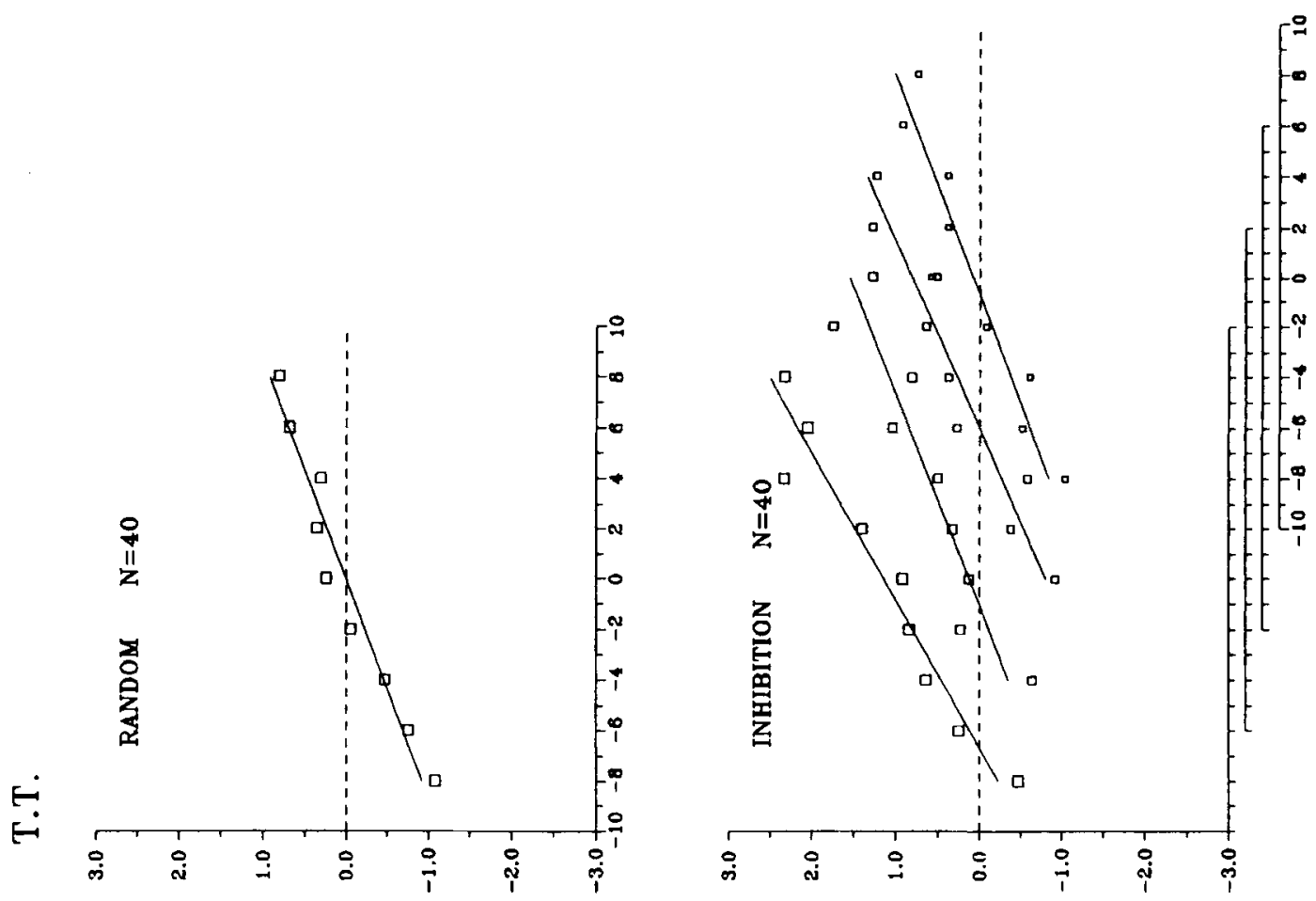

1
2
0
0
0
0
0
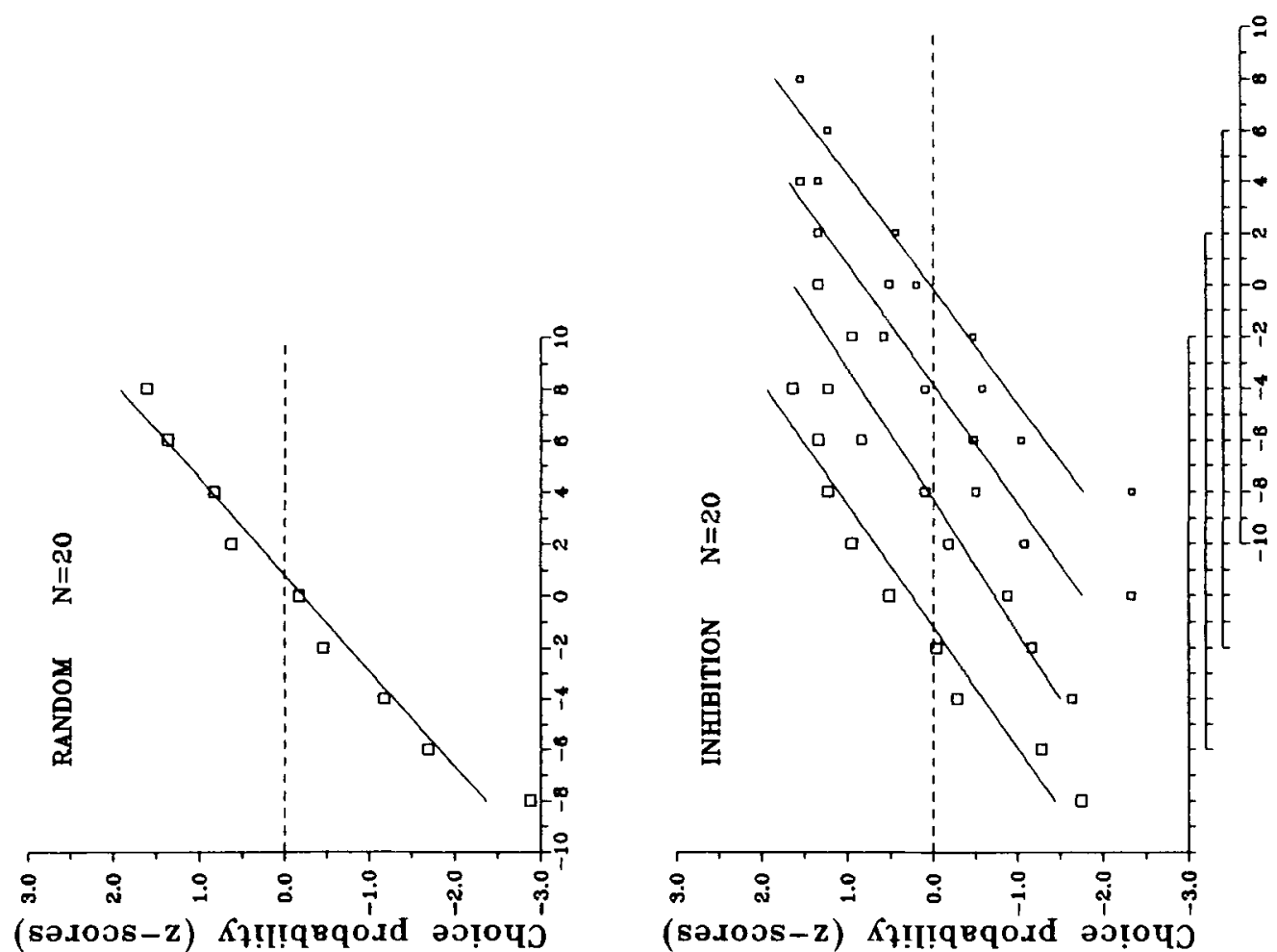

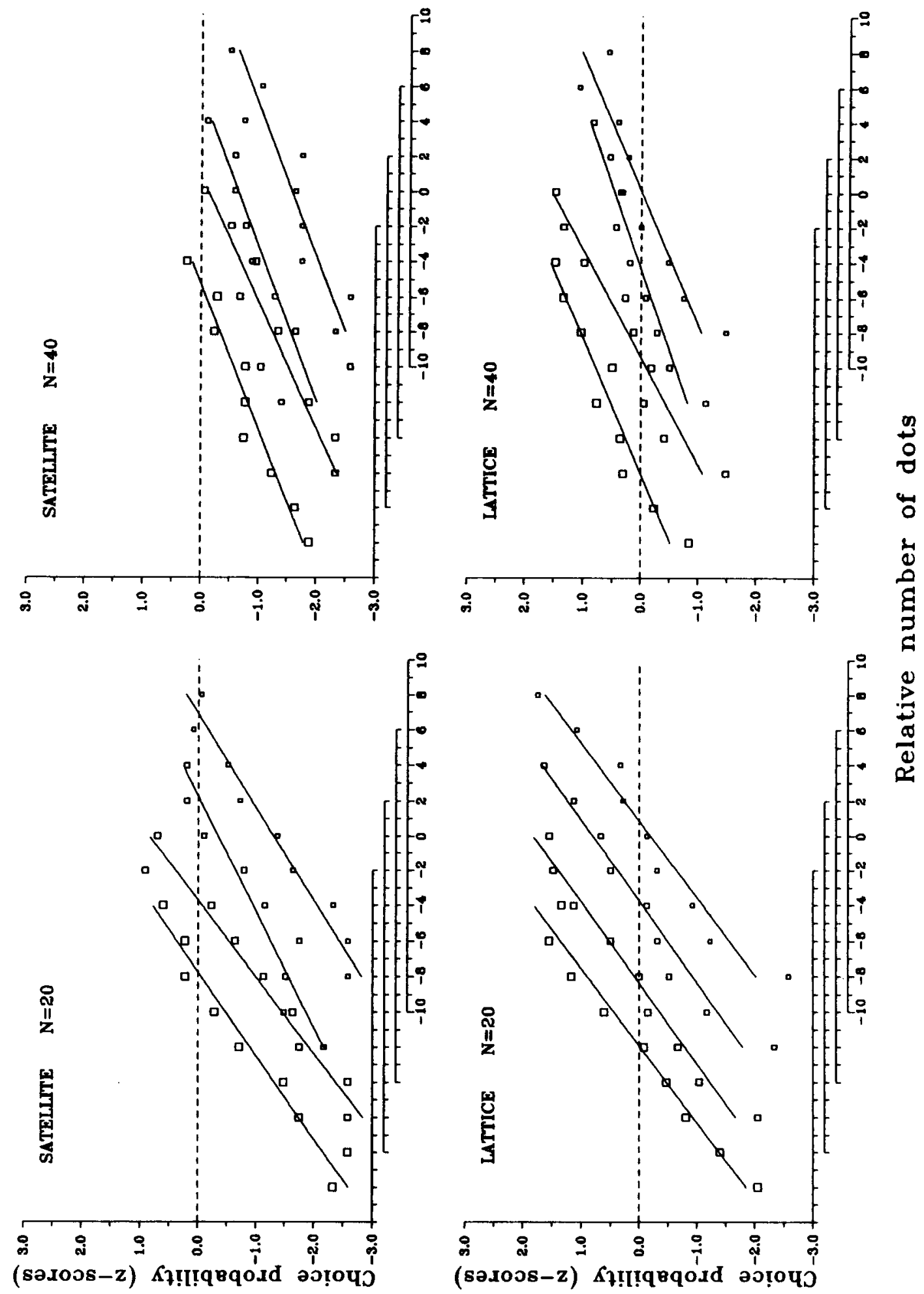

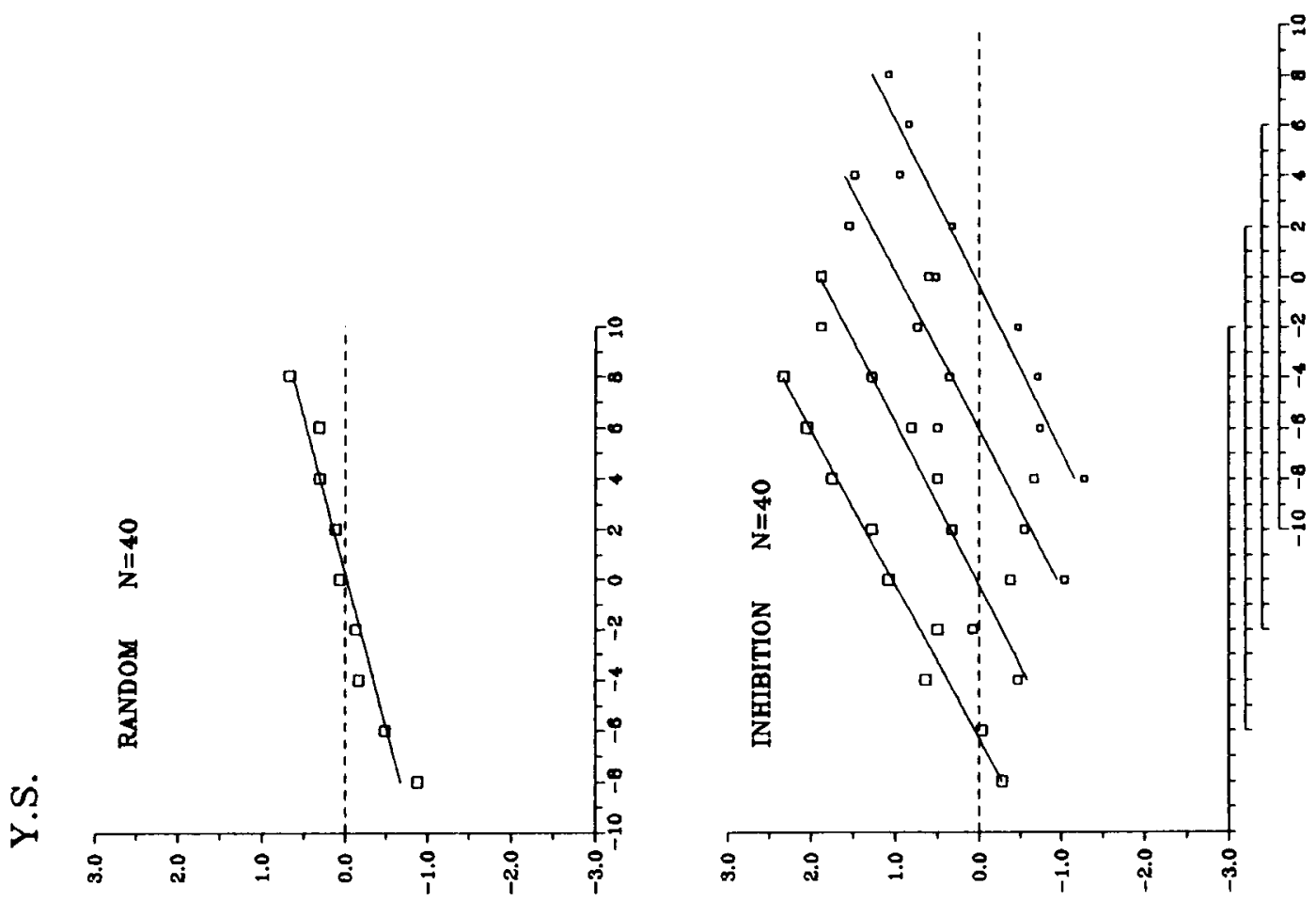

04
0
0
010
0
0
0
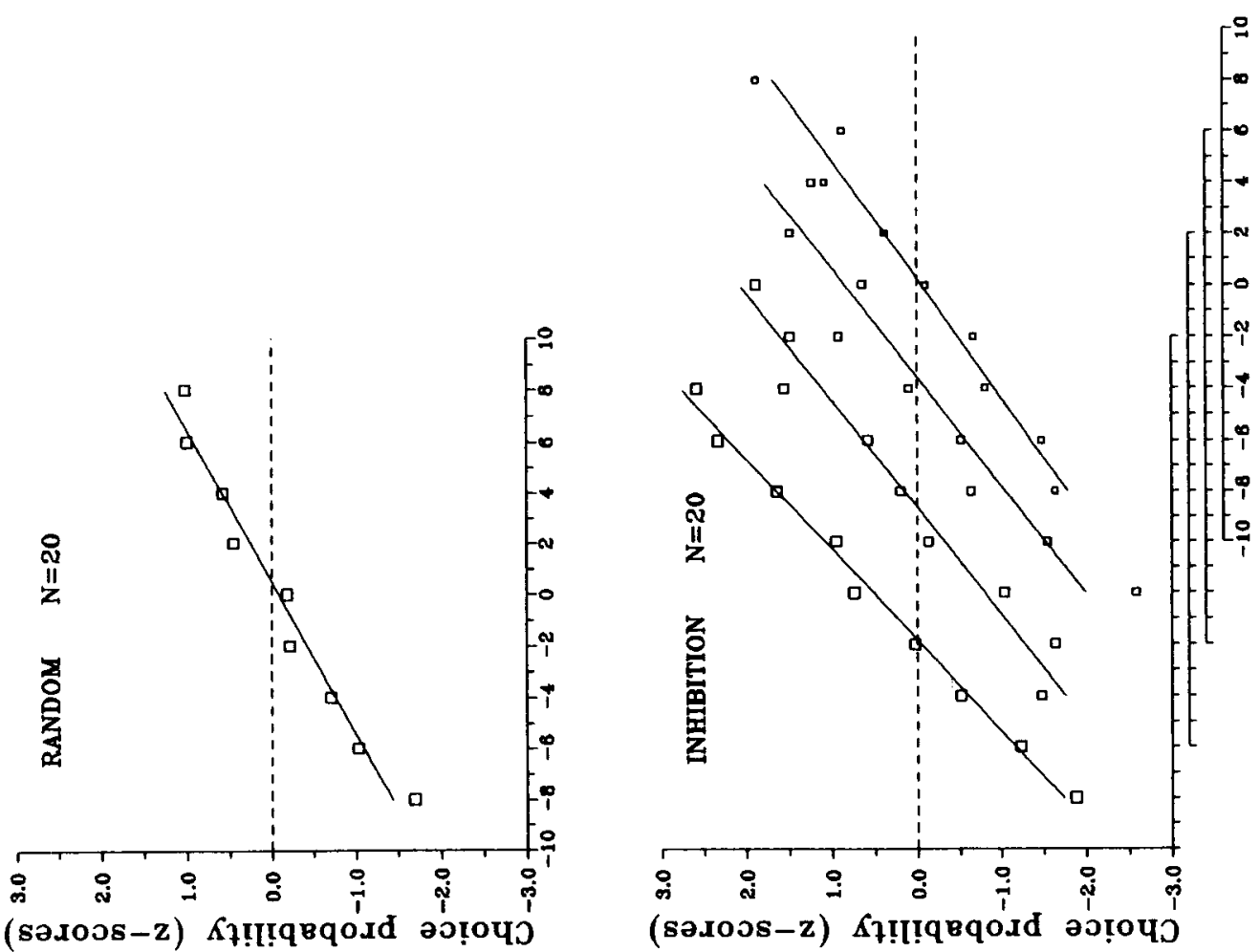

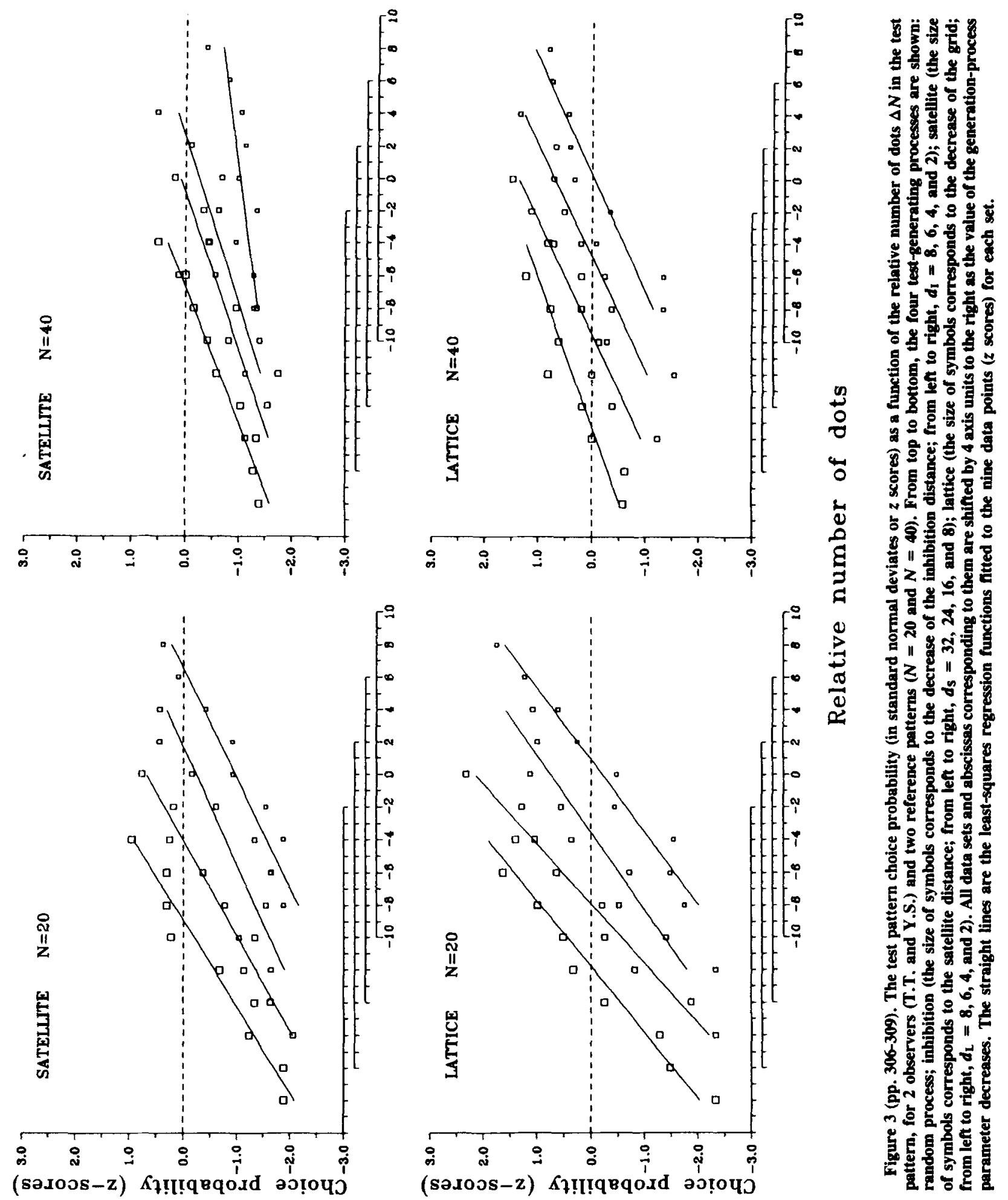

젼

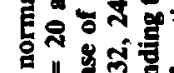

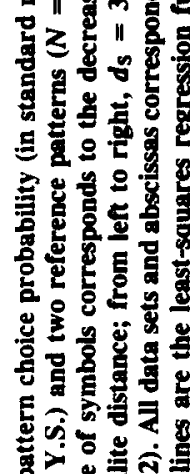

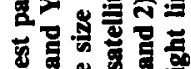

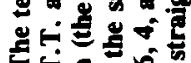

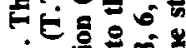

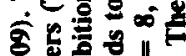

के 령

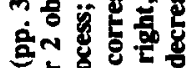

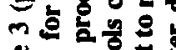

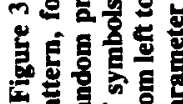

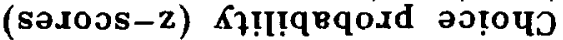


relative number of dots $\Delta N$ in the test pattern, for subjects T.T. (Figure 3A) and Y.S. (Figure 3B), for each reference pattern $(N=20, N=40)$.

In Figure 3, the best-fitting least-squares linear regressions are represented by straight lines. The fits are generally quite good, and the Pearson product-moment correlation coefficient exceeds .9 in most cases (Table 1). The intercept of the regression line with the zero level (broken lines) indicates the point of subjective equality (PSE). The slope of the regression line indicates the differential sensitivity of visual number, and it can be used to determine the just noticeable difference (JND) - that is, the increase or decrease of the relative number $\Delta N$ that is required by subjects to correctly discriminate the test pattern from the reference pattern in $75 \%$ of the cases. The numerical values of the PSE and JND are given in Table 1.

The slope is steeper and the JND smaller for the smaller reference pattern $(N=20)$ than for the larger one $(N=40)$. On the average, approximately 3.0 (T.T.) or 3.4 (Y.S.) extra dots are just noticeably different from 20 reference dots, and 5.3 (T.T.) or 6.2 (Y.S.) from 40 reference dots. The corresponding Weber fractions, $k$ $(k=\mathrm{JND} / N)$, are $.152(N=20)$ and $.133(N=40)$ for T.T. and .168 and .155, respectively, for Y.S. These values are typical of numerosity discrimination experiments. The Weber fraction for numerosity discrimination has been estimated to be $.134(N=20)$ and .128 $(N=40)$ (Newman, 1974); .162 $(N=8 \div 30)$ (van Oeffelen \& Vos, 1982); and .124 $(N=25)$ (Krueger, 1984). The observed decrease of the Weber fraction with reference number has also been reported by most researchers (Burgess \& Barlow, 1983; Krueger, 1984; Newman, 1974).

The PSE for the random distribution is generally moderately positive, reflecting an asymmetry of the psychometric function; numerosity decrements are more noticeable than increments (van Oeffelen \& Vos, 1982). By contrast, the normal distribution function used for approximation is symmetric around zero. The PSEs for the inhibition patterns are generally negative; these patterns look more numerous than does the reference pattern with the same size. As the inhibitory distance $d_{\mathrm{I}}$ around each dot increases, this tendency increases (see Table 1). The numerosity overestimation of the inhibitory patterns is also more conspicuous for patterns with a larger number of dots $(N=40)$. The same type of overestimation is observed for the lattice patterns. As the grid step $d_{\mathrm{L}}$ of the invisible lattice increases, the apparent number of dots increases, especially when $N=40$. The dot patterns generated by the inhibition and lattice processes are similar, in that each dot is surrounded by an exclusionary territory within which no other dot can occur. Thus, both numerosity illusions can be ascribed to inhibitory overestimation.

The appearance of satellite patterns is different from that of inhibitory patterns. All psychometric functions are
Table 1

Numerical Values of Best Linear Fit Shown in Figure 3

\begin{tabular}{|c|c|c|c|c|c|c|}
\hline \multirow[b]{2}{*}{ Process } & \multicolumn{3}{|c|}{$N=20$} & \multicolumn{3}{|c|}{$N=40$} \\
\hline & $r$ & PSE & JND & $r$ & PSE & JND \\
\hline \multicolumn{7}{|c|}{ SUBJECT T.T. } \\
\hline \multicolumn{7}{|c|}{ Random } \\
\hline & .98 & 0.8 & 2.5 & .97 & -0.1 & 5.8 \\
\hline \multicolumn{7}{|c|}{ Inhibition } \\
\hline$d_{\mathrm{I}}=2$ & .96 & -0.2 & 3.0 & .93 & -0.7 & 5.8 \\
\hline$d_{1}=4$ & .97 & 0.1 & 3.1 & .96 & -2.0 & 5.0 \\
\hline$d_{1}=6$ & .97 & -0.3 & 3.4 & .92 & -5.0 & 5.6 \\
\hline$d_{\mathrm{I}}=8$ & .97 & -1.2 & 3.2 & .97 & -6.7 & 3.9 \\
\hline \multicolumn{7}{|c|}{ Satellite } \\
\hline$d_{\mathrm{s}}=8$ & .98 & 6.9 & 3.6 & .92 & 13.4 & 5.8 \\
\hline$d_{\mathrm{S}}=16$ & .95 & 6.2 & 4.4 & .86 & 9.5 & 5.9 \\
\hline$d_{\mathrm{S}}=24$ & .98 & 4.3 & 2.9 & .95 & 8.9 & 4.8 \\
\hline$d_{\mathrm{s}}=32$ & .98 & 4.2 & 3.2 & .97 & 6.7 & 5.5 \\
\hline \multicolumn{7}{|c|}{ Lattice } \\
\hline$d_{\mathrm{L}}=2$ & .97 & 0.8 & 3.0 & .93 & 0.0 & 5.2 \\
\hline$d_{\mathrm{L}}=4$ & .98 & 0.3 & 3.1 & .96 & -0.4 & 6.3 \\
\hline$d_{\mathrm{L}}=6$ & .98 & -0.4 & 3.1 & .96 & -1.4 & 4.1 \\
\hline$d_{\mathrm{L}}=8$ & .98 & 0.1 & 2.9 & .96 & -4.0 & 5.2 \\
\hline \multicolumn{7}{|c|}{ SUBJECT Y.S. } \\
\hline \multicolumn{7}{|c|}{ Random } \\
\hline & .98 & 0.5 & 4.0 & .97 & 0.2 & 8.2 \\
\hline \multicolumn{7}{|c|}{ Inhibition } \\
\hline$d_{1}=2$ & .99 & 0.2 & 3.1 & .96 & -0.4 & 4.4 \\
\hline$d_{I}=4$ & .96 & 0.4 & 2.9 & .95 & -2.1 & 4.2 \\
\hline$d_{1}=6$ & .98 & -0.7 & 2.8 & .96 & -4.2 & 4.3 \\
\hline$d_{1}=8$ & .99 & -1.8 & 2.5 & .98 & -6.3 & 4.1 \\
\hline \multicolumn{7}{|c|}{ Satellite } \\
\hline$d_{\mathrm{S}}=8$ & .96 & 6.5 & 4.5 & .78 & 24.3 & 15.9 \\
\hline$d_{\mathrm{S}}=16$ & .88 & 5.7 & 4.9 & .88 & 6.5 & 6.9 \\
\hline$d_{\mathrm{s}}=24$ & .98 & 4.0 & 3.9 & .83 & 7.1 & 6.5 \\
\hline$d_{\mathrm{s}}=32$ & .97 & 3.1 & 3.6 & .98 & 5.3 & 5.6 \\
\hline \multicolumn{7}{|c|}{ Lattice } \\
\hline$d_{\mathrm{L}}=2$ & .98 & 0.9 & 3.0 & .93 & 0.3 & 4.9 \\
\hline$d_{\mathrm{L}}=4$ & .95 & 0.5 & 3.2 & .94 & -0.8 & 4.7 \\
\hline$d_{\mathrm{L}}=6$ & .99 & 0.1 & 2.3 & .96 & -1.5 & 4.7 \\
\hline$d_{\mathrm{L}}=8$ & .98 & 0.2 & 2.7 & .92 & -3.3 & 6.2 \\
\hline
\end{tabular}

Note $-r=$ Pearson product-moment correlation. PSE = point of subjective equality. JND $=$ just noticeable difference.

shifted downward; the larger positive PSEs indicate that the satellite patterns are underestimated, and the PSE values decrease with satellite distance $d_{\mathrm{s}}$. Thus, the underestimation peaks when the satellite dot is placed within a $16 \times 16$ pixel rectangle $\left(d_{\mathrm{s}}=8\right)$ centered at the parent dot, and the expected distance between a parent dot and its satellite is 5.7 pixels. In this case, the function generally lies below the dotted lines (see Figure 3); that is, the apparent reduction in numerosity for patterns composed from 48 dots $(\Delta N=8)$ exceeds 8 dots.

In general, the results agree with data reported by Ginsburg and Goldstein (1987). The inhibitory patterns, which have a more regular appearance, were judged to be more numerous than were the completely random patterns, 
which in turn were judged to be more numerous than the satellite patterns, which have a more clustered (or aggregated) appearance.

\section{THE OCCUPANCY MODEL}

We believe that the main thrust of the Vos et al. (1988) model is correct-perceived numerosity depends on the area of the stimulus field apparently occupied by a constellation of dots. Although the brightness of each dot is precisely localized, its influence is spread over a much wider area. Our criticism was directed against the particular CODE algorithm whose predictions contradict the manifest appearance of some dot patterns (see Figure 1). According to the CODE algorithm, the width of the dispersion function depends on the distance between each dot and its nearest neighbor. This appears to be incorrect, at least for the estimation of numerosity. Our analysis demonstrates that the spread function of each dot is independent of the spatial proximity of dots. The impact of any dot upon its surroundings appears to be constant, irrespective of the spatial arrangement of the dots. According to the proposed occupancy model, each dot occupies a circular territory of radius $R$ centered at the dot. If two dots are less than distance $R$ apart, their individual territories overlap, and the contribution of the pair of dots is reduced proportionally to the size of the overlap of their occupied territories.

In Figure 4, the separation between the dots in the lefthand pair (A) is shorter than $R$, and, consequently, their individual spaces overlap. In contrast, the distance between the right-hand dots (B) exceeds the occupancy radius $R$, and their occupied territories are isolated. It is obvious that the area covered by both dots is larger in the second case than it is in the first one. The occupancy model postulates that the occupancy index (the total area of the stimulus field occupied by dots) provides the basis for the judgment of numerosity. If two patterns are presented, the hemifield that is apparently occupied by
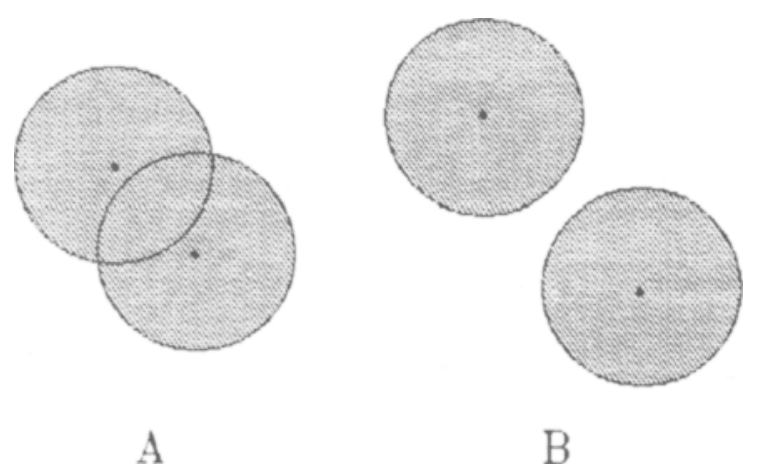

Figure 4. The basic tenet of the occupancy model: (A) If two dots are close enough to each other (less than radius $R$ ), the territories they occupy overlap. (B) If the interdot distance exceeds the radius $R$, there is no intersection between the two occupied territories. The impression of numerosity is posited to be proportional to the total area occupied by dots. This area is langer for Case $B$ than for Case $\mathbf{A}$. more dots will, as a rule, be chosen as being more numerous.

The proposed model seems intuitively plausible. If a plane area $A^{2}$ is randomly bombarded by $N$ circles with radius $R$, the expected proportion of the plane covered by the circles is $1-\exp \left(-\pi R^{2} N / A^{2}\right)$ (Schachter \& Ahuja, 1979). The shape of the figure is not critical. The proportion of the plane covered by figures will be the same for any collection of congruent convex figures, each of them with the same area $\pi R^{2}$, randomly distributed over the plane. The proportion of the plane covered by circles can be smaller or larger for pattern-generation processes differing from completely random bombardment. In an inhibitory pattern, circles overlap less than they do in a random pattern, in which they in turn overlap less than they do in a satellite pattern. Consequently, given the amount of territory occupied, the apparent numerosity of inhibitory patterns ought to be overestimated and that of satellite patterns underestimated, compared with that of entirely random patterns.

Let us now describe the occupancy model more formally. When the test and reference patterns are presented, the observer is presumed to compute the occupancy indices for both of them. The pattern with the larger $o c$ cupancy value is chosen as being more numerous. Thus, the sign of the relative occupancy index (test-reference) determines the observer's numerosity judgments. The occupancy index of stochastic patterns is a random variable. Two samples of the same dot pattern type will in general have different occupancy values. Given a large enough sample size, the distribution will be close to normal, and it is sufficient to know only the mean $\mu$ and the variance $\sigma^{2}$ of the occupancy index distribution. Given these two values, $\mu$ and $\sigma^{2}$, for both members of the test and reference pattern pairs, it is easy to find from them the test pattern choice probability:

$$
P=\Phi \frac{\mu_{\mathrm{T}}-\mu_{\mathrm{R}}}{\sqrt{\sigma_{\mathrm{T}}^{2}+\sigma_{\mathrm{R}}^{2}}}
$$

where $\Phi$ stands for the standard normal integral, and the indexes $T$ and $R$ stand for the test and reference patterns, respectively. It is cumbersome to obtain analytical estimates of the mean and variance of the numerosity index for each random pattern-generation procedure. Therefore, we generated 100 samples of each pattern type and determined directly for each pair of test and reference patterns its relative occupancy index $\mu_{T}-\mu_{R}$ and its standard deviation $\sqrt{ }\left(\sigma_{\mathrm{T}}^{2}+\sigma_{\mathrm{R}}^{2}\right)$. If numerosity judgments are indeed based on the relative occupancy index, the test pattern choice probability can be directly derived from this objectively measured property of the pair of dot patterns. One of the simplest ways to test this prediction is to plot the relative occupancy indices as standard normal deviates $\left(\mu_{T}-\mu_{R}\right) / \sqrt{ }\left(\sigma_{T}^{2}+\sigma_{R}^{2}\right)$ against the test pattern choice probabilities also expressed in standard normal deviates ( $z$ scores). If the hypothesis is correct, all 234 data points can be described by a single linear function. 


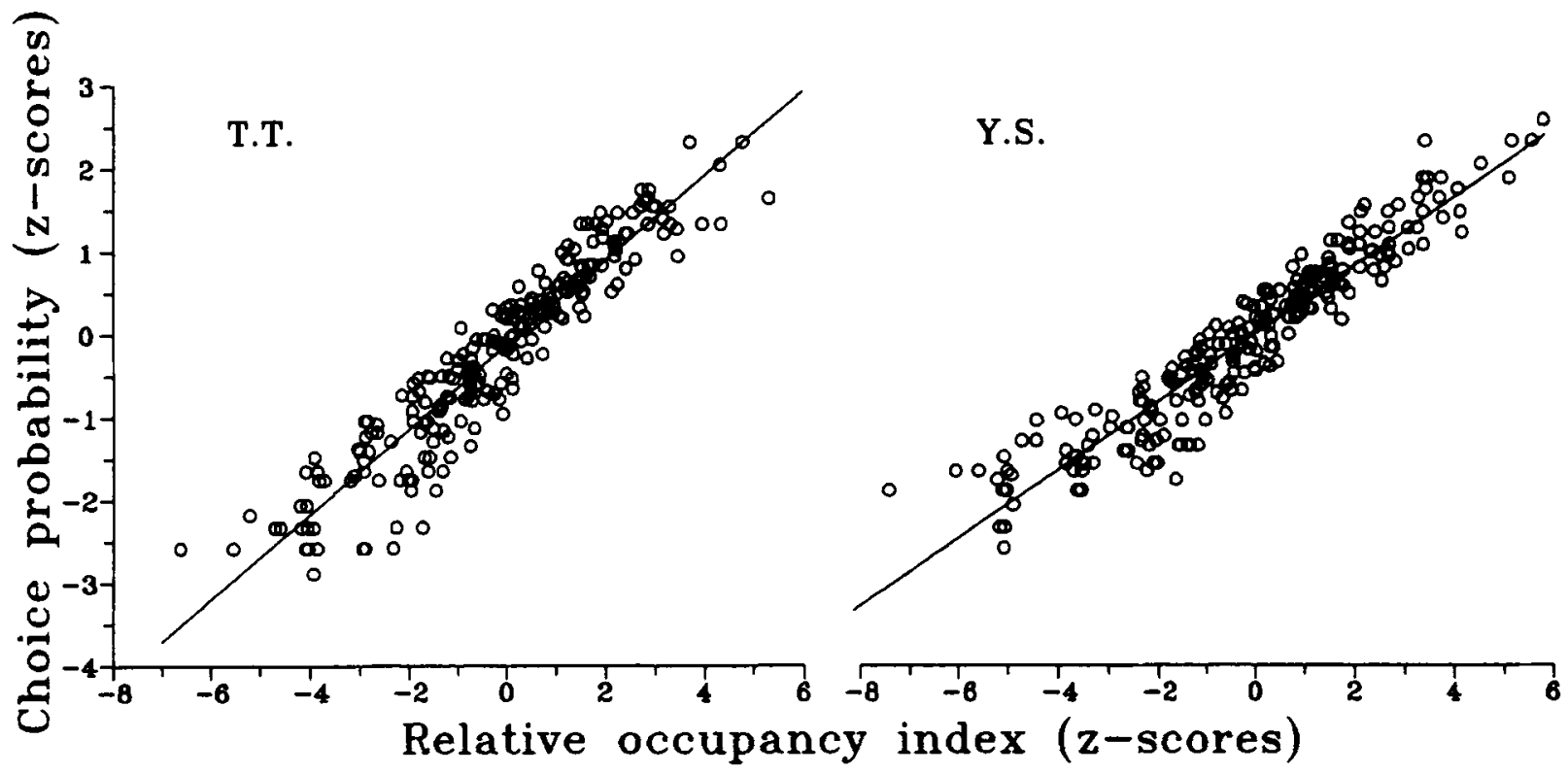

Figure 5. The test pattern choice probability ( $z$ scores) predicted from the normalized relative occupancy index for 234 pairs of dot patterns.

Figure 5 shows the test pattern choice probability as a function of the relative occupancy index (both expressed in $z$ scores) for the subjects T.T. (left panel) and Y.S. (right panel). The proposed occupancy model has only one free parameter: the occupancy radius $R$. The relative occupancy index depends on the value of this parameter, which was estimated using a grid search. The optimal value $R$ (i.e., the value of the occupancy radius $R$ that produced the maximum Pearson product-moment correlation coefficient in the grid search) was 11 pixels or approximately $22^{\prime}$ of arc for the observer T.T. and 9 pixels or approximately $18^{\prime}$ of arc for the observer Y.S. The correlation coefficient for the best-fitting functions (Figure 5) were highly significant in both cases ( $r=.940$ and $r=.946$, respectively).

It might seem that the choice of a parameter describing random-dot patterns is rather arbitrary and that some other spatial statistics might predict the observer's choices just as well as the occupancy index. For comparison, we chose the two spatial statistics most frequently used to describe spatial point processes: the nearest neighbor distance and the number of elements per probe area (Diggle, 1977, 1983; Ripley, 1981). The procedure used for computing these two statistics was analogous to that used for computing the relative occupancy index. For each pair of patterns, the relative mean nearest neighbor distance divided by its standard deviation was found. The correlations with the observer's choices were $r=.779$ (T.T.) and $r=.712$ (Y.S.), which are considerably smaller than those obtained in the case of the occupancy index. In order to estimate the number of dots per probe area, each hemifield was divided into $10 \times 10$ square cells. Again, the mean and variance of the number of dots per probe area were found, and then the difference between the test and reference patterns normalized by their summary standard deviation was found. The correlations between psychometrical values and these spatial statistics were $r=.798$ (T.T.) and $r=.838$ (Y.S.). Thus, in both cases, the fit is inferior to that of the occupancy index. The perceived numerosity depends on neither the nearest neighbor distance nor the number of elements per probe area.

\section{DISCUSSION}

It is very satisfying that such a simple and completely deterministic model with a single free parameter, the occupancy radius $R$, is able to predict the numerosity choice probabilities so well. The proposed occupancy model accounts for approximately $88 \%-89 \%$ of the variance in the data-which is remarkable, considering the relatively large random scatter of empirical psychometric functions. According to the proposed explanation, every dot has an impact on its proximal surroundings, occupying a territory around it within a fixed radius $R$. The estimated radius of occupancy is about $20^{\prime}$ of visual angle (in absolute terms) or about $2.2 \%$ of the total stimulus area (in relative terms), but the exact value seems not to be very critical. On the basis of the present data alone, it is impossible to say in which domain, absolute or relative, the occupancy radius is defined. Also, other experiments are needed to determine whether the occupancy radius $R$ is a constant or depends on some stimulus property. In the present study, the same fixed value of $R$ was assigned to all dots, for each observer. It is more plausible to assume that $R$ is a random variable reflecting the internal noise 
of the observer. If one takes into account this internal noise, the predictions of the occupancy model could be even more accurate.

What is the psychological meaning of the influence a dot has upon its neighborhood in the radius $R$ ? According to the occupancy model, two proximate dots have less total impact on the numerosity decision than do two spatially distant ones. Perhaps this is simply another way of saying that a dot is inhibited (or masked) by another nearby dot. It is well known that the contrast sensitivity of a dot is decreased by the presence of another dot at some eccentricity from the first one (Westheimer, 1967). But it is rather doubtful that the decrease in visual number due to proximity of dots can be explained entirely by contrast desensitization, which spreads no farther than about 10' of arc. Moreover, as Mulligan and MacLeod (1988) recently demonstrated, an increase in dot density makes lit dots appear not dimmer, as desensitization would predict, but brighter. The brightness appears to be integrated over a distance of approximately $30^{\prime}$ of arc in radius. However, there is no information about how this brightness enhancement might be related to apparent numerosity. We suspect that the occupancy radius reflects some more fundamental properties of spatial vision. It seems that the occupancy model can be easily reinterpreted in terms of the existing models of spatial vision. In particular, the MIRAGE algorithm (Watt, 1988; Watt \& Morgan, 1985) assumes the convolution of the input luminance profile with spatial filters of different sizes. The output of each filter, having symmetrical center-surround organization, is further split into two separate components, the zero-bounded positive $T^{+}$and negative $T^{-}$portions of the filter output. At an appropriate spatial scale, the territory occupied by the $T^{+}$signal appears to be a good approximation of the occupancy index proposed in this study.

How can the total area occupied by dots be estimated? It is known that the figures of equal area but different shape are judged as differing in area (cf. Anastasi, 1936; Smith, 1969). The perceived area depends not only on the physical area but on other stimulus attributes, such as the convex perimeter or the maximal linear extent of the pattern as well. If we propose that the numerosity is estimated on the basis of the phenomenal impression of the area occupied, we have to devise an additional theory to explain the perception of the area of irregular shapes. To avoid such a complication, perceived numerosity and the area apparently occupied by dots must not be considered as two separate perceptual attributes. The area occupied by dots is experienced, or simply seen, as numerosity.

The occupancy model has some notable advantages over the other explanations of perceived numerosity proposed so far. It appears to be a sufficiently general model, because it was tested on a set of 234 random-dot patterns differing from each other both in their number and in the spatial configuration of their elements. The occupancy model can also be characterized as a formal model with a minimal number of parameters not specified in strictly quantitative terms. In this respect, it can be compared only with the CODE algorithm that was applied to the analysis of various numerosity illusions (Vos et al., 1988). Unfortunately, the computational complexity of the CODE algorithm makes the direct comparison of these two models difficult. There were 14,400 positions in each hemifield and 1,404,000 dots in 234 different stimulus pairs repeated at least 100 times each in our experiment. Thus, the computation of about $2 \times 10^{10}$ values of a normal distribution function is required.

In fact, there may be no need for a rigorous test, because the CODE model obviously fails to predict the perceived numerosity of patterns shown in Figure 1, which are very similar to those used in the present experiment. In any case, the occupancy model gives much more plausible numerosity estimates of these patterns. First, the occupancy index clearly distinguishes random patterns with different numbers of dots (Figures 1C-1D): 19.4\% $(N=10), 37.1 \%(N=20)$, and $50.9 \%$ occupancy $(N=30)$. Recall that the CODE algorithm regards them all as about equal in perceived numerosity. Next, the $40-$ dot satellite pattern (Figure 1A) is predicted by its occupancy index (45.6\%) to appear to be more numerous than 20 but less numerous than 30 randomly distributed dots. This estimate is definitely better than the CODE prophecy that the satellite pattern appears to be even less numerous than the 10 uncorrelated dots in Figure 1C. In addition, the occupancy model predicts the direction of many known numerosity illusions at least as well as does the CODE algorithm. In particular, dots arranged in a circular pattern must appear to be less numerous than the same number of randomly distributed dots (Taves, 1941), because the occupied territories of two neighboring dots overlap more in a circle than in a random distribution. The "regular-random illusion" reported by Ginsburg (1976) is in fact just one of many possible inhibitory patterns, whose numerosity overestimation has already been explained in terms of the occupancy model.

The value of a new theory is revealed, in particular, by its ability to anticipate unknown phenomena and to provide better explanations of already known ones. For example, Burgess and Barlow (1983) demonstrated that two regular (inhibitory) distributions of dots are discriminated more precisely than two completely random arrays of dots. If, for example, the number of dots increases from 40 to 46 , then the proportion of the plane covered by a completely random distribution of circles with a radius of 10 pixels increases approximately $5.1 \%$. At the same time, the proportion of the covered plane increases approximately $6.7 \%$ for the inhibitory distribution with an inhibition distance of 8 pixels. Consequently, the same increase in the number of dots is more salient in a regular (inhibitory) distribution than in a completely random array of dots. Thus, the occupancy model can explain why numerosity is discriminated better in regular distributions. Next, Burgess and Barlow (1983) found that the JND varies approximately as the 0.75 power of $N$ (i.e., 
JND $=k N^{0.75}$, where $k$ is the coefficient of proportionality). They write:

\begin{abstract}
We cannot find any easy explanation for the exponent of about 0.7 in this relationship. Since the variance of the number of dots in each small area is Poisson distributed and therefore proportional to dot density, it would be relatively easy to explain an exponent of 0.5 : indeed such a relationship could be explained in a number of ways. There are also some grounds for expecting an exponent of unity, since this is the prediction of Weber's Law. All that can be said is that the empirical value lies between these "explainable" values. (p. 816)
\end{abstract}

The occupancy index is not a linear function of the dot number $N$. For a Poisson distribution of dots, the expected proportion of a unit plane covered by the circles of radius $R$ is $1-\exp \left(-\pi R^{2} N\right)$. We found that the power relation between the number of randomly distributed dots $(N=12$ to 48 ) and the proportion of the plane $S$ covered by circles $(R=10)$ was well described by a power function $(r=.990)$ with the exponent of $0.731\left(S=k N^{0.731}\right)$. However, the latter is not a constant, because it depends on the occupancy radius $R$. In this particular case, the size of the JND is approximately a linear function of the occupancy index $(0.75 / 0.731 \approx 1)$. In other words, an exponent of unity (Weber's law) may not be expected between the number of reference dots per se but the areas they appear to occupy in the stimulus plane. This can also explain why psychophysical scales for numerosity are typically highly compressive functions of $N$ (Indow \& Ida, 1977; Krueger, 1984); the perceived numerosity is not directly connected with the dot number but with an attribute that itself is a compressive function of the dot number.

\section{REFERENCES}

ALLIK, J. (1989). Is a unified psychophysical law realistic? Behavioral \& Brain Sciences, 12, 267-268.

ANASTASI, A. (1936). The estimation of area. Journal of General Psychology, 14, 201-225.

Bevan, W. Maier, R., \& Helson, H. (1963). The influence of context upon the estimation of number. American Journal of Psychology, 76, 464-469.

BiNET, A. (1890). La perception des longueurs et des nombres chez quelques petits enfants. Revue Philosophique de la France et de l'Étranger, 30, 68-81.
Burgess, A., Barlow, H. B. (1983). The precision of mumerosity discrimination in arrays of random dots. Vision Research. 23, 811-820.

DIGGLE, P. J. (1977). The detection of random heterogeneity in plant populations. Biometrics, 33, 390-394.

DIGGLE, P. J. (1983). Statistical analysis of spatial patterns. London: Academic Press.

FrITH, C. D., \& FrITH, U. (1972). The solitaire illusion: An illusion of numerosity. Perception \& Psychophysics, 11, 409-410.

GinSBURG, N. (1976). Effect of item arrangement on perceived numerosity: Randomness vs regularity. Perceptual \& Motor Skills, 43, 663-668

GinsbuRG, N., \& Goldstein, S. R. (1987). Measurement of visual cluster. American Journal of Psychology, 100, 193-203.

Indow, T., \& IDA, M. (1977). Scaling of dot numerosity. Perception \& Psychophysics, 22, 265-276.

Krueger, L. E. (1972). Perceived numerosity. Perception \& Psychophysics, 11, 5-9.

Krueger, L. E. (1984). Perceived numerosity: A comparison of magnitude production, magnitude estimation, and discrimination judgments. Perception \& Psychophysics, 35, 536-542

Mulligan, J. B., \& MacLeod, D. I. A. (1988). Reciprocity between luminance and dot density in the perception of brightness. Vision Research, 28, 503-519.

Newman, C. V. (1974). Detection of differences between visual textures with varying number of dots. Bulletin of the Psychonomic Society, 4, 201-202.

Ponzo, M. (1928). Unteilstäuschungen über Mengen. Archiv fiur die gesamte Psychologie, 65, 129-162.

RIPLEY, B. D. (1977). Modelling spatial patterns (with discussion). Journal of the Royal Statistical Society, 39B, 172-212.

RipLEY, B. D. (1981). Spatial statistics. New York: Wiley.

SCHACHTER, B., \& AHUSA, N. (1979). Random pattern generation processes. Computer Graphics \& Image Processing, 10, 95-114.

SMITH, J. P. (1969). The effects of figural shape on the perception of area. Perception \& Psychophysics, 5, 49-52.

TAves, E. H. (1941). Two mechanisms for the perception of visual numerousness. Archives of Psychology, 37, 1-47.

van Oeffelen, M. P., \& Vos, P. G. (1982). A probabilistic model for the discrimination of visual number. Perception \& Psychophysics, 32, 163-170.

van Oeffelen, M. P., \& Vos, P. G. (1983). An algorithm for pattern description on the level of relative proximity. Pattern Recognition, 16, 341-348.

Vos, P. G., van Oeffelen, M. P., Tibosch, H. J., Allik, J. (1988). Area-numerosity interactions. Psychological Research, 50, 148-150.

WATT, R. J. (1988). Visual processing: Computational, psychophysical, and cognitive research. Hove, U.K.: Erlbaum.

WATt, R. J., \& MORGAN, M. J. (1985). A theory of the primitive spatial code in human vision. Vision Research, 25, 1661-1674.

WESTHEIMER, G. (1967). Spatial interaction in human cone vision. Journal of Physiology, 190, 139-154.

(Manuscript received April 23, 1990; revision accepted for publication October $17,1990$. ) 